\title{
Technicolor
}

\section{DYNAMICAL ELECTROWEAK SYMMETRY BREAKING}

Revised October 2001 by R.S. Chivukula and M. Narain (Boston University) and J. Womersley (Fermilab).

In theories of dynamical electroweak symmetry breaking, the electroweak interactions are broken to electromagnetism by the vacuum expectation value of a fermion bilinear. These theories may thereby avoid the introduction of fundamental scalar particles, of which we have no examples in nature. In this note, we review the status of experimental searches for the particles predicted in technicolor, topcolor, and related models. The limits from these searches are summarized in Table 1.

\section{Technicolor}

The earliest models $[1,2]$ of dynamical electroweak symmetry breaking [3] include a new non-abelian gauge theory ("technicolor") and additional massless fermions ("technifermions") which feel this new force. The global chiral symmetry of the fermions is spontaneously broken by the formation of a technifermion condensate, just as the approximate chiral $S U(2) \times S U(2)$ symmetry in QCD is broken down to $S U(2)$ isospin by the formation of a quark condensate. If the quantum numbers of the technifermions are chosen correctly (e.g. by choosing technifermions in the fundamental representation of an $\mathrm{SU}(N)$ technicolor gauge group, with the left-handed technifermions being weak doublets and the right-handed ones weak singlets) this condensate can break the electroweak interactions down to electromagnetism.

The breaking of the global chiral symmetries implies the existence of Goldstone bosons, the "technipions" $\left(\pi_{T}\right)$. Through

HTTP://PDG.LBL.GOV Page $1 \quad$ Created: 6/13/2002 13:08 
the Higgs mechanism, three of the Goldstone bosons become the longitudinal components of the $W$ and $Z$, and the weak gauge bosons acquire a mass proportional to the technipion decay constant (the analog of $f_{\pi}$ in QCD). The quantum numbers and masses of any remaining technipions are model dependent. There may be technipions which are colored (octets and triplets) as well as those carrying electroweak quantum numbers, and some color-singlet technipions are too light $[4,5]$ unless additional sources of chiral-symmetry breaking are introduced. The next lightest technicolor resonances are expected to be the analogs of the vector mesons in QCD. The technivector mesons can also have color and electroweak quantum numbers and, for a theory with a small number of technifermions, are expected to have a mass in the TeV range [6].

While technicolor chiral symmetry breaking can give mass to the $W$ and $Z$ particles, additional interactions must be introduced to produce the masses of the standard model fermions. The most thoroughly studied mechanism for this invokes "extended technicolor" (ETC) gauge interactions [4,7]. In ETC, technicolor, color and flavor are embedded into a larger gauge group which is broken to technicolor and color at an energy scale of 100s to 1000s of TeV. The massive gauge bosons associated with this breaking mediate transitions between quarks/leptons and technifermions, giving rise to the couplings necessary to produce fermion masses. The ETC gauge bosons also mediate transitions among technifermions themselves, leading to interactions which can explicitly break unwanted chiral symmetries and raise the masses of any light technipions. The ETC interactions connecting technifermions to quarks/leptons also mediate technipion decays to ordinary fermion pairs. Since

HTTP://PDG.LBL.GOV Page 2 Created: 6/13/2002 13:08 
Table 1: Summary of the mass limits. Symbols are defined in the text.

\begin{tabular}{|c|c|c|c|}
\hline Process & Excluded mass range & Decay channels & Ref. \\
\hline$p \bar{p} \rightarrow \rho_{T} \rightarrow W \pi_{T}$ & $\begin{array}{c}170<m_{\rho_{T}}<190 \mathrm{GeV} \\
\quad \text { for } m_{\pi_{T}} \approx m_{\rho_{T}} / 2\end{array}$ & $\begin{array}{c}\rho_{T} \rightarrow W \pi_{T} \\
\pi_{T}^{0} \rightarrow b \bar{b} \pi_{T}^{ \pm} \rightarrow b \bar{c}\end{array}$ & $\bar{c}^{[15]}$ \\
\hline$p \bar{p} \rightarrow \omega_{T} \rightarrow \gamma \pi_{T}$ & $\begin{array}{c}140<m_{\omega_{T}}<290 \mathrm{GeV} \\
\text { for } m_{\pi_{T}} \approx m_{\omega_{T}} / 3 \\
\text { and } M_{T}=100 \mathrm{GeV}\end{array}$ & $\begin{array}{c}\omega_{T} \rightarrow \gamma \pi_{T} \\
\pi_{T}^{0} \rightarrow b \bar{b} \\
\pi_{T}^{ \pm} \rightarrow b \bar{c}\end{array}$ & [17] \\
\hline$\overline{p \bar{p}} \rightarrow \omega_{T} / \rho_{T}$ & $\begin{array}{c}m_{\omega_{T}}=m_{\rho_{T}}<203 \mathrm{GeV} \\
\text { for } m_{\omega_{T}}<m_{\pi_{T}}+m_{W} \\
\quad \text { or } M_{T}>200 \mathrm{GeV}\end{array}$ & $\omega_{T} / \rho_{T} \rightarrow \ell^{+} \ell^{-}$ & {$[18]$} \\
\hline$e^{+} e^{-} \rightarrow \omega_{T} / \rho_{T}$ & $\begin{array}{c}90<m_{\rho_{T}}<206.7 \mathrm{GeV} \\
m_{\pi_{T}}<79.8 \mathrm{GeV}\end{array}$ & $\begin{array}{l}\rho_{T} \rightarrow W W \\
W \pi_{T}, \pi_{T} \pi_{T} \\
\gamma \pi_{T}, \text { hadrons }\end{array}$ & {$[20]$} \\
\hline$p \bar{p} \rightarrow \rho_{T 8}$ & $260<m_{\rho_{T 8}}<480 \mathrm{GeV}$ & $\rho_{T 8} \rightarrow q \bar{q}, g g$ & {$[23]$} \\
\hline $\begin{aligned} p \bar{p} & \rightarrow \rho_{T 8} \\
& \rightarrow \pi_{L Q} \pi_{L Q}\end{aligned}$ & $\begin{aligned} m_{\rho_{T 8}} & <510 \mathrm{GeV} \\
m_{\rho_{T 8}} & <600 \mathrm{GeV} \\
m_{\rho_{T 8}} & <465 \mathrm{GeV}\end{aligned}$ & $\begin{array}{l}\pi_{L Q} \rightarrow c \nu \\
\pi_{L Q} \rightarrow b \nu \\
\pi_{L Q} \rightarrow \tau q\end{array}$ & $\begin{array}{l}{[26]} \\
{[26]} \\
{[25]}\end{array}$ \\
\hline$p \bar{p} \rightarrow g_{t}$ & $\begin{array}{c}0.3<m_{g_{t}}<0.6 \mathrm{TeV} \\
\text { for } 0.3 m_{g_{t}}<\Gamma<0.7 m_{g_{t}}\end{array}$ & $g_{t} \rightarrow b \bar{b}$ & {$[31]$} \\
\hline$\overline{p \bar{p}} \rightarrow Z^{\prime}$ & $\begin{array}{l}m_{Z^{\prime}}<480 \mathrm{GeV} \\
\text { for } \Gamma=0.012 m_{Z^{\prime}} \\
m_{Z^{\prime}}<780 \mathrm{GeV} \\
\text { for } \Gamma=0.04 m_{Z^{\prime}}\end{array}$ & $Z^{\prime} \rightarrow t \bar{t}$ & {$[32]$} \\
\hline
\end{tabular}

these interactions are responsible for fermion masses, one generally expects technipions to decay to the heaviest fermions kinematically allowed (though this need not hold in all models).

In addition to quark masses, ETC interactions must also give rise to quark mixing. One expects, therefore, that there are 


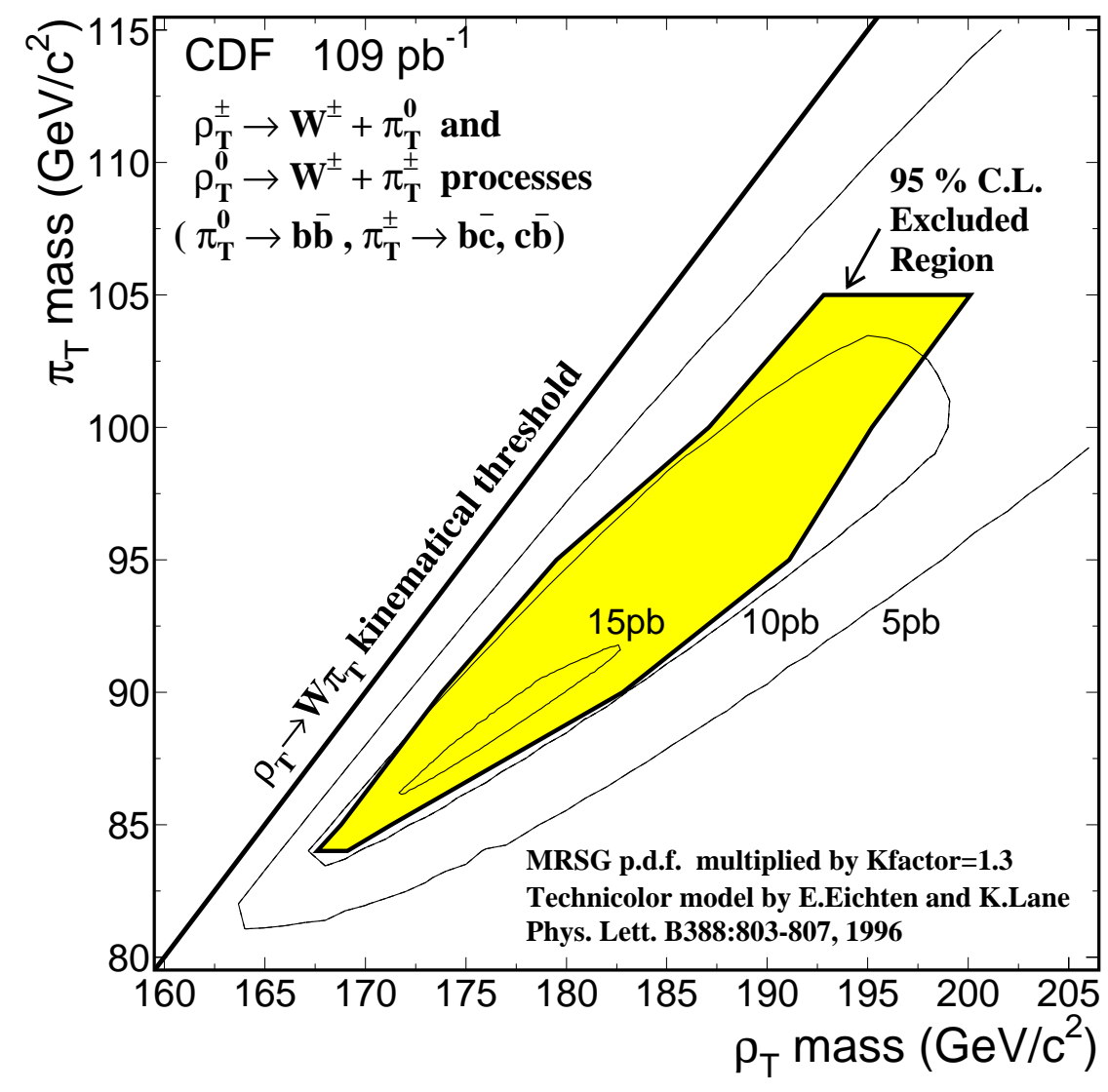

Figure 1: 95\% CL exclusion region [15] for a light technirho decaying to $W^{ \pm}$and a $\pi_{T}$, and in which the $\pi_{T}$ decays to two jets including at least one $b$ quark.

ETC interactions coupling quarks of the same charge from different generations. A stringent limit on these flavor-changing neutral current interactions comes from $K^{0}-\bar{K}^{0}$ mixing [4]. These force the scale of ETC breaking and the corresponding ETC gauge boson masses to be in the 100-1000 TeV range (at HTTP://PDG.LBL.GOV Page $4 \quad$ Created: 6/13/2002 13:08 
least insofar as ETC interactions of first two generations are concerned). To obtain quark and technipion masses that are large enough then requires an enhancement of the technifermion condensate over that expected naively by scaling from QCD. Such an enhancement can occur if the technicolor gauge coupling runs very slowly, or "walks" [8]. Many technifermions typically are needed to make the TC coupling walk, implying that the technicolor scale and, in particular, the technivector mesons may be much lighter than $1 \mathrm{TeV}[3,9]$. It should also be noted that there is no reliable calculation of electroweak parameters in a walking technicolor theory, and the values of precisely measured electroweak quantities [10] cannot directly be used to constrain the models.

In existing colliders, technivector mesons are dominantly produced when an off-shell standard model gauge-boson "resonates" into a technivector meson with the same quantum numbers [11]. The technivector mesons may then decay, in analogy with $\rho \rightarrow \pi \pi$, to pairs of technipions. However, in walking technicolor the technipion masses may be increased to the point that the decay of a technirho to pairs of technipions is kinematically forbidden [9]. In this case the decay to a technipion and a longitudinally polarized weak boson (an "eaten" Goldstone boson) may be preferred, and the technivector meson would be very narrow. Alternatively, the technivector may also decay, in analogy with the decay $\rho \rightarrow \pi \gamma$, to a technipion plus a photon, gluon, or transversely polarized weak gauge boson. Finally, in analogy with the decay $\rho \rightarrow e^{+} e^{-}$, the technivector meson may resonate back to an off-shell gluon or electroweak gauge boson, leading to a decay into a pair of leptons, quarks, or gluons.

When comparing the various results presented in this review, one should be aware that the more recent analyses HTTP://PDG.LBL.GOV Page 5 Created: 6/13/2002 13:08 
$[18,20,21]$ make use of newer calculations [12] of technihadron production and decay, as implemented in PYTHIA version 6.126 and higher [13]. The results obtained with older cross section calculations are not always directly comparable.

If the dominant decay mode of the technirho is $W_{L} \pi_{T}$, promising signal channels [14] are $\rho_{T}^{ \pm} \rightarrow W^{ \pm} \pi_{T}^{0}$ and $\rho_{T}^{0} \rightarrow$ $W^{ \pm} \pi_{T}^{\mp}$. If we assume that the technipions decay to $b \bar{b}$ (neutral) and $b \bar{c}$ (charged), then both channels yield a signal of $W(\ell \nu)+2$ jets, with one or more heavy flavor tags. The CDF collaboration has carried out a search in this final state [15] based on Run I data and using PYTHIA [16] version 6.1 for the signal simulation. The results are shown in Figure 1. We see that the search is sensitive to $\sigma \cdot B \gtrsim 10 \mathrm{pb}$ and that roughly $170<m_{\rho_{T}}<190 \mathrm{GeV}$ is excluded at the $95 \%$ confidence level, for $m_{\pi_{T}} \approx m_{\rho_{T}} / 2$. For an integrated luminosity of $2 \mathrm{fb}^{-1}$ in Run II, the $5 \sigma$ discovery reach extends to $m_{\rho_{T}}=210 \mathrm{GeV}$ and $m_{\pi_{T}}=110 \mathrm{GeV}$, while the $95 \%$ exclusion sensitivity will extend to $m_{\rho_{T}}=250 \mathrm{GeV}$ and $m_{\pi_{T}}=145 \mathrm{GeV}$.

$\mathrm{CDF}$ has also searched [17] for the process $\omega_{T}^{0} \rightarrow \gamma \pi_{T}^{0}$, yielding a signal of a hard photon plus two jets, with one or more heavy flavor tags. The sensitivity to $\sigma \cdot B$ is of order $1 \mathrm{pb}$. The excluded region is shown in Fig. 2 and is roughly $140<m_{\omega_{T}}<290 \mathrm{GeV}$ at the $95 \%$ level, for $m_{\pi_{T}} \approx m_{\omega_{T}} / 3$. The analysis assumes four technicolors, $Q_{D}=Q_{U}-1=\frac{1}{3}$ and $M_{T}=100 \mathrm{GeV} / c^{2}$. Here $Q_{U}$ and $Q_{D}$ are the charges of the lightest technifermion doublet and $M_{T}$ is a dimensionful parameter, of order $100 \mathrm{GeV} / c^{2}$, which controls the rate of $\rho_{T}, \omega_{T} \rightarrow \gamma \pi_{T}$.

Both DØ [18] and CDF [19] have searched for low-scale technicolor resonances $\rho_{T}$ and $\omega_{T}$ decaying to dileptons, using inclusive $e^{+} e^{-}$(both experiments) and $\mu^{+} \mu^{-}$(CDF) samples from

HTTP://PDG.LBL.GOV Page $6 \quad$ Created: 6/13/2002 13:08 


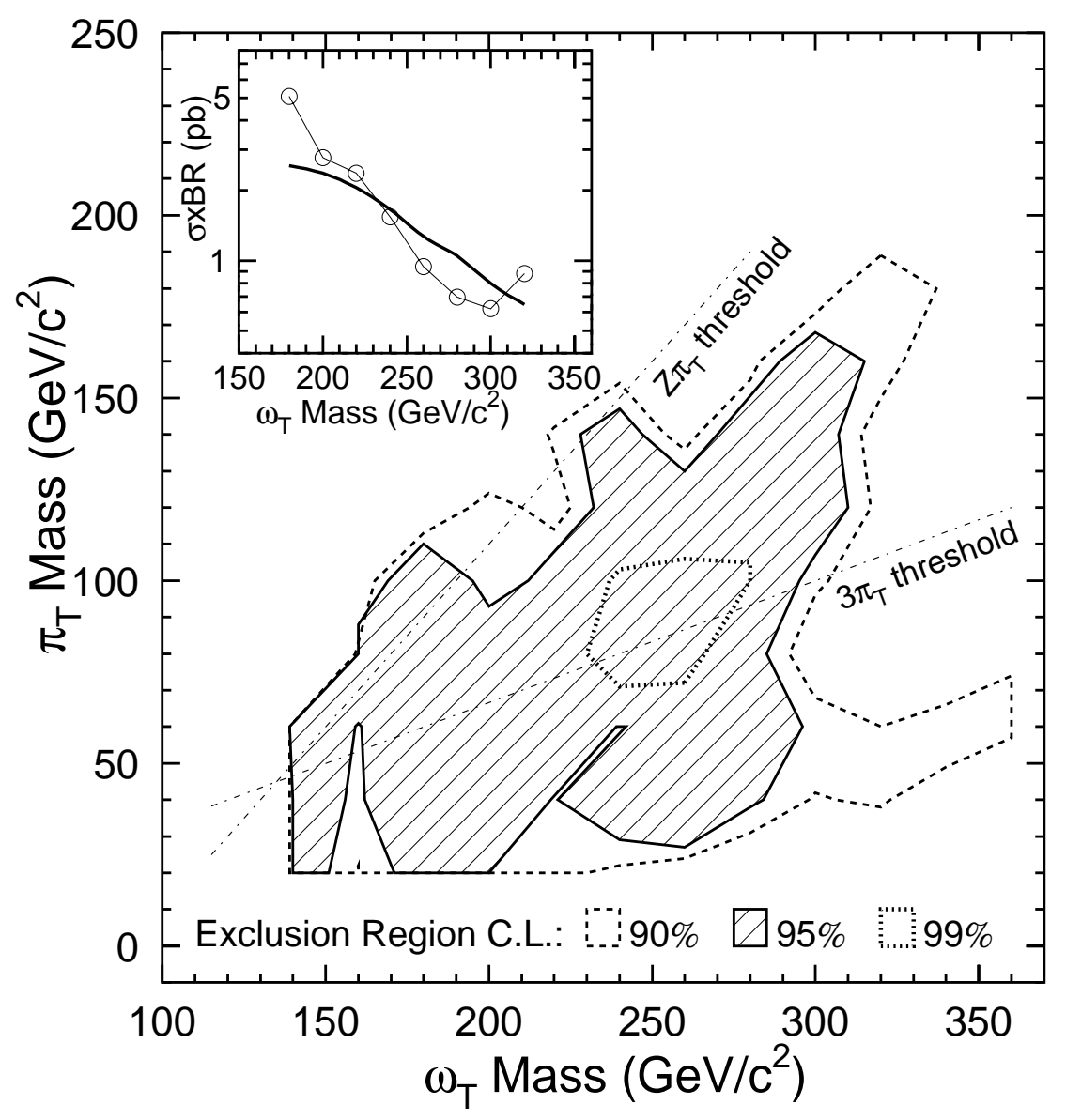

Figure 2: $95 \%$ CL exclusion region [17] for a light techniomega decaying to $\gamma$ and a $\pi_{T}$, and in which the $\pi_{T}$ decays to two jets including at least one $b$ quark. (Inset: cross section limit for $m_{\pi_{T}}=120 \mathrm{GeV}$.)

Run I. In the search, the $\rho_{T}$ and $\omega_{T}$ are assumed to be degenerate in mass. The absence of structure in the dilepton invariant mass distribution is then used to set limits. Those from $D \varnothing$

HTTP://PDG.LBL.GOV Page $7 \quad$ Created: 6/13/2002 13:08 


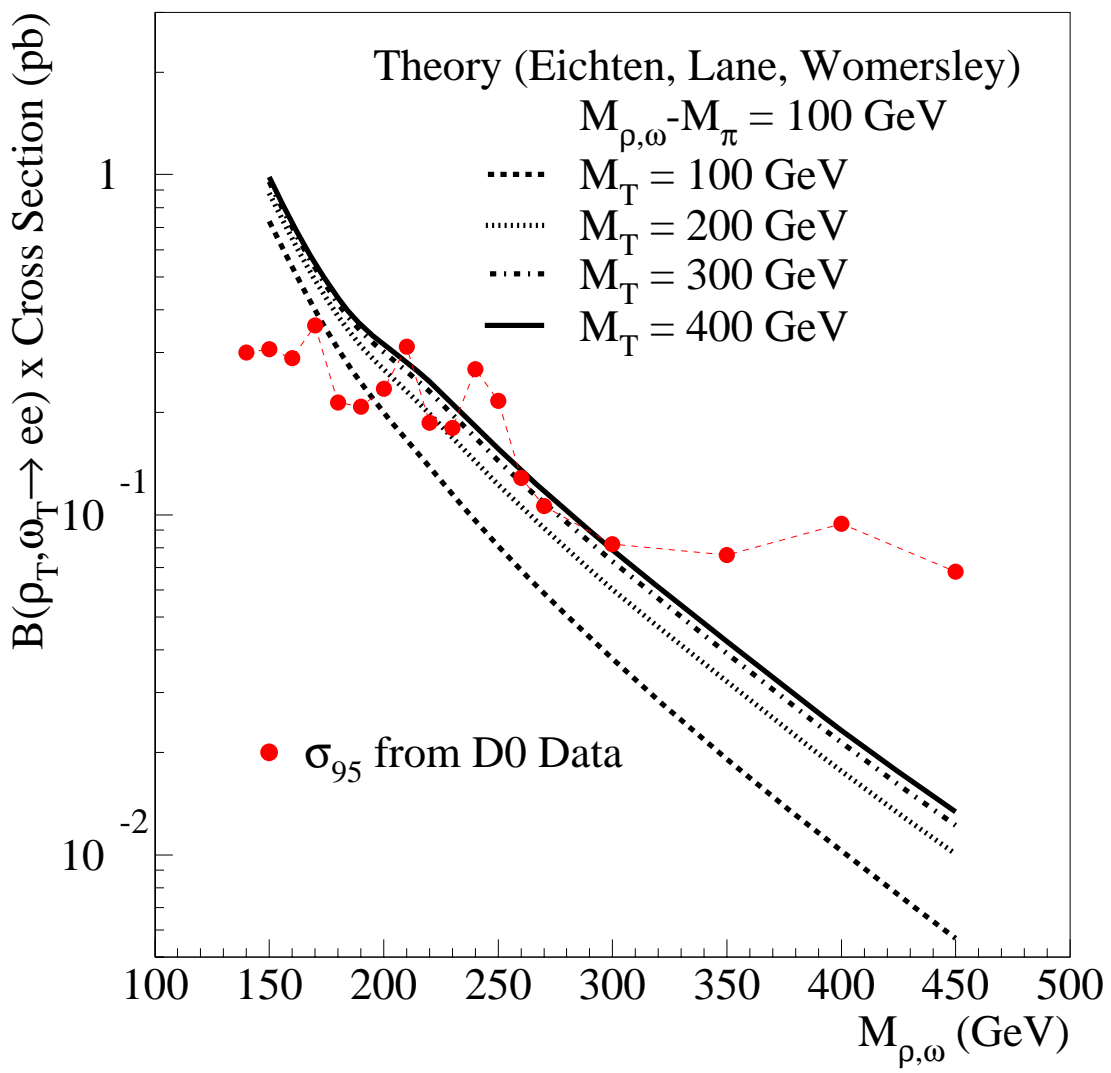

Figure 3: 95\% CL cross-section limit [18] for a light techniomega and a light technirho decaying to $\ell^{+} \ell^{-}$.

are slightly more restrictive. Masses $m_{\rho_{T}}=m_{\omega_{T}} \lesssim 200 \mathrm{GeV}$ are excluded, provided either $m_{\rho_{T}}<m_{\pi_{T}}+m_{W}$, or $M_{T}>200 \mathrm{GeV}$ (as shown in Fig. 3). With $2 \mathrm{fb}^{-1}$ of data in Run II, the sensitivity will extend to $m_{\rho_{T}}=m_{\omega_{T}} \approx 500 \mathrm{GeV}$.

HTTP://PDG.LBL.GOV Page $8 \quad$ Created: 6/13/2002 13:08 


\section{DELPHI}

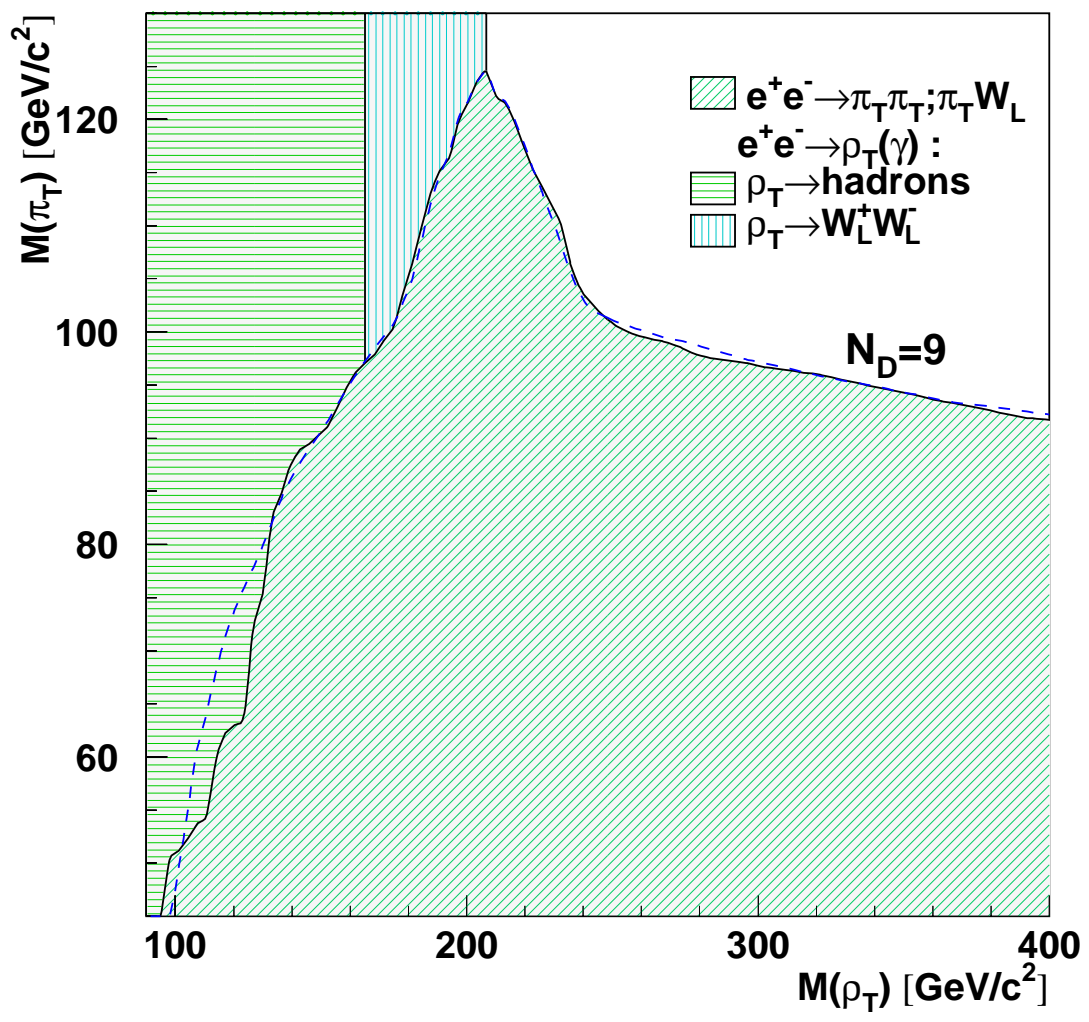

Figure 4: 95\% CL exclusion region [20] in the technirho-technipion mass plane obtained from searches by the DELPHI collaboration at LEP 2, for nine technifermion doublets. The dashed line shows the expected limit for the 4 -jet analysis.

DELPHI [20] has reported a search for technicolor production in $452 \mathrm{pb}^{-1}$ of $e^{+} e^{-}$data taken between 192 and 208

HTTP://PDG.LBL.GOV Page $9 \quad$ Created: 6/13/2002 13:08 
$\mathrm{GeV}$. The analysis combines searches for $e^{+} e^{-} \rightarrow \rho_{T}(\gamma)$ with $\rho_{T} \rightarrow W_{L} W_{L}, \rho_{T} \rightarrow$ hadrons $\left(\pi_{T} \pi_{T}\right.$ or $\left.q \bar{q}\right), \rho_{T} \rightarrow \pi_{T} \gamma$, and $e^{+} e^{-} \rightarrow \rho_{T}^{*} \rightarrow W_{L} \pi_{T}$ or $\pi_{T} \pi_{T}$. Technirho masses in the range $90<m_{\rho_{T}}<206.7 \mathrm{GeV}$ are excluded, while technipion masses $m_{\pi_{T}}<79.8(89.1) \mathrm{GeV}$ are ruled out for 2 (9) technifermion doublets. L3 [21] reported a similar search, with somewhat less restrictive results, in 1999.

Searches have also been carried out at the Tevatron for colored technihadron resonances [22,23]. CDF has used a search for structure in the dijet invariant mass spectrum to set limits on a color-octet technirho $\rho_{T 8}$ produced by an off-shell gluon and decaying to two real quarks or gluons. As shown in Fig. 5 masses $260<m_{\rho_{T 8}}<480 \mathrm{GeV}$ are excluded; in Run II the limits will improve to cover the whole mass range up to about $0.8 \mathrm{TeV}[24]$.

The CDF second and third-generation leptoquark searches (see Refs. [25,26]) have also been interpreted in terms of the complementary $\rho_{T 8}$ decay mode: $p \bar{p} \rightarrow \rho_{T 8} \rightarrow \pi_{L Q} \pi_{L Q}$. Here $\pi_{L Q}$ denotes a color-triplet technipion carrying both color and lepton number, assumed to decay to $b \nu$ or $c \nu[26]$ or to a $\tau$ plus a quark [25]. The searches exclude technirho masses $m_{\rho_{T 8}}$ less than $510 \mathrm{GeV}\left(\pi_{L Q} \rightarrow c \nu\right), 600 \mathrm{GeV}\left(\pi_{L Q} \rightarrow b \nu\right)$, and $465 \mathrm{GeV}$ $\left(\pi_{L Q} \rightarrow \tau q\right)$ for technipion masses up to $m_{\rho_{T 8}} / 2$. Figure 6 shows the $\pi_{L Q} \rightarrow b \nu$ exclusion region. (Leptoquark masses $m_{\pi_{L Q}}$ less than $123 \mathrm{GeV}(c \nu), 148 \mathrm{GeV}(b \nu)$, and $99 \mathrm{GeV}$ $(\tau q)$ are already ruled out by standard continuum-production leptoquark searches).

Recently, it has been demonstrated that there is substantial uncertainty in the theoretical estimate of the $\rho_{T 8}$ production cross section at the Tevatron and that the cross section may be as much as an order of magnitude lower than the naive vector meson dominance estimate [27]. To establish the range HTTP://PDG.LBL.GOV Page $10 \quad$ Created: 6/13/2002 13:08 


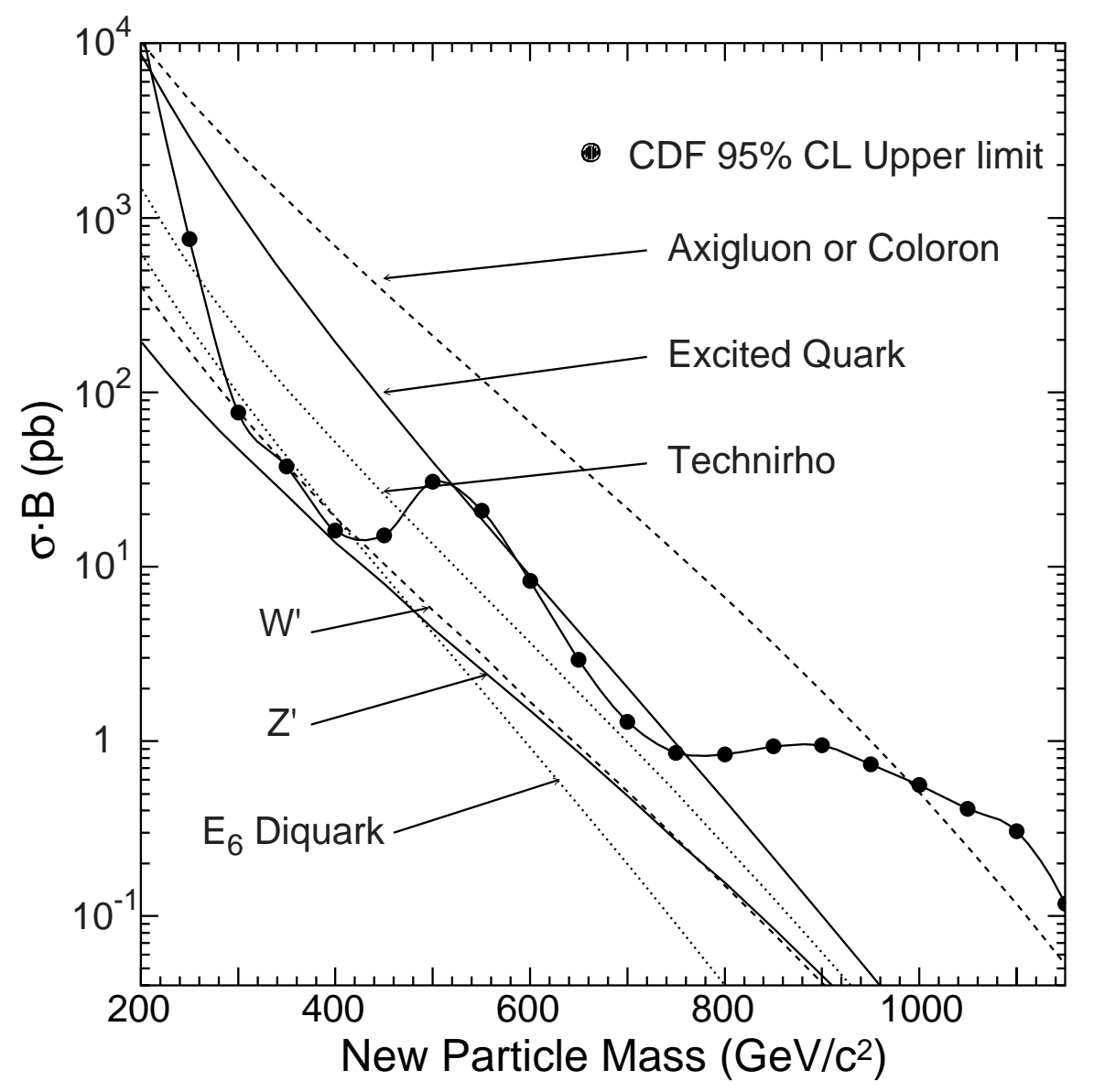

Figure 5: 95\% CL Cross-section limits [23] for a technirho decaying to two jets at the Tevatron.

of allowed masses, these limits will need to be redone with a reduced theoretical cross section.

\section{Top Condensate and Related Models}

The top quark is much heavier than other fermions and must be more strongly coupled to the symmetry-breaking sector. It 


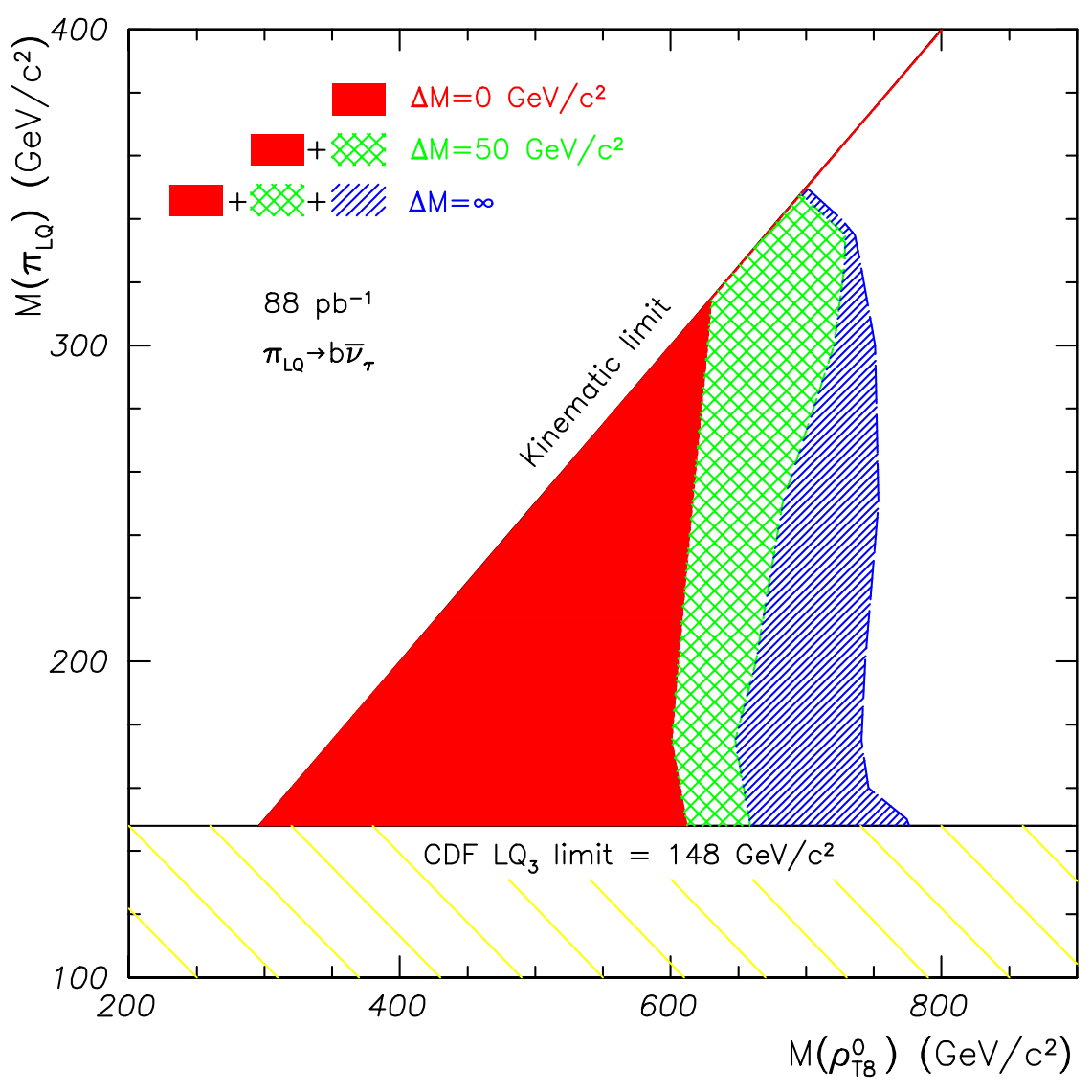

Figure 6: 95\% CL exclusion region [26] in the technirho-technipion mass plane for pair produced technipions, with leptoquark couplings, decaying to $b \nu$.

is natural to consider whether some or all of electroweaksymmetry breaking is due to a condensate of top quarks [3,28]. Top-quark condensation alone, without additional fermions, seems to produce a top-quark mass larger [29] than observed 

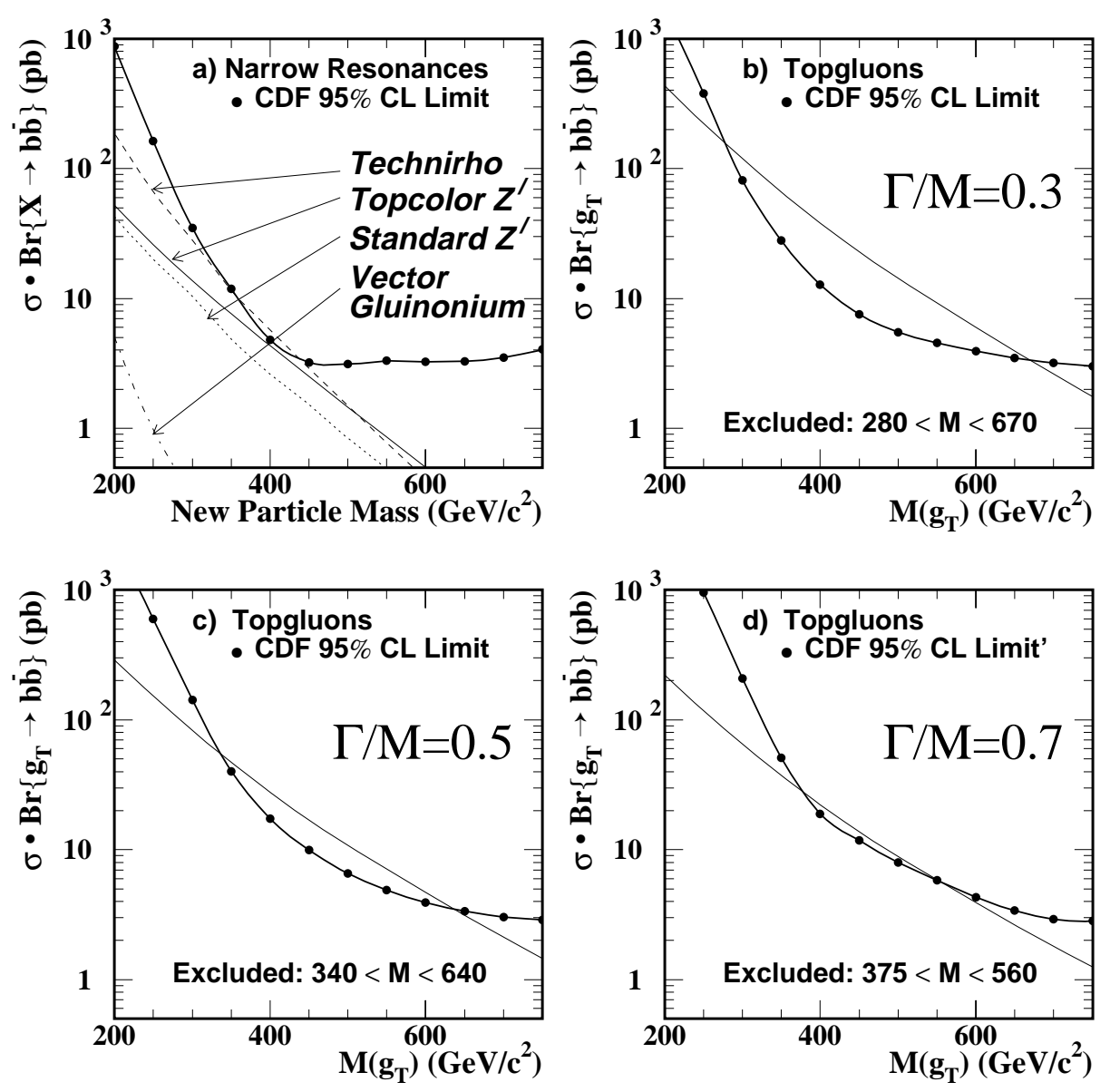

Figure 7: Tevatron limits [31] on new particles decaying to $b \bar{b}$ : narrow resonances and topgluons for various widths.

experimentally, and is therefore not favored. Topcolor assisted technicolor [30] combines technicolor and top-condensation. In addition to technicolor, which provides the bulk of electroweak symmetry breaking, top-condensation and the top quark mass 


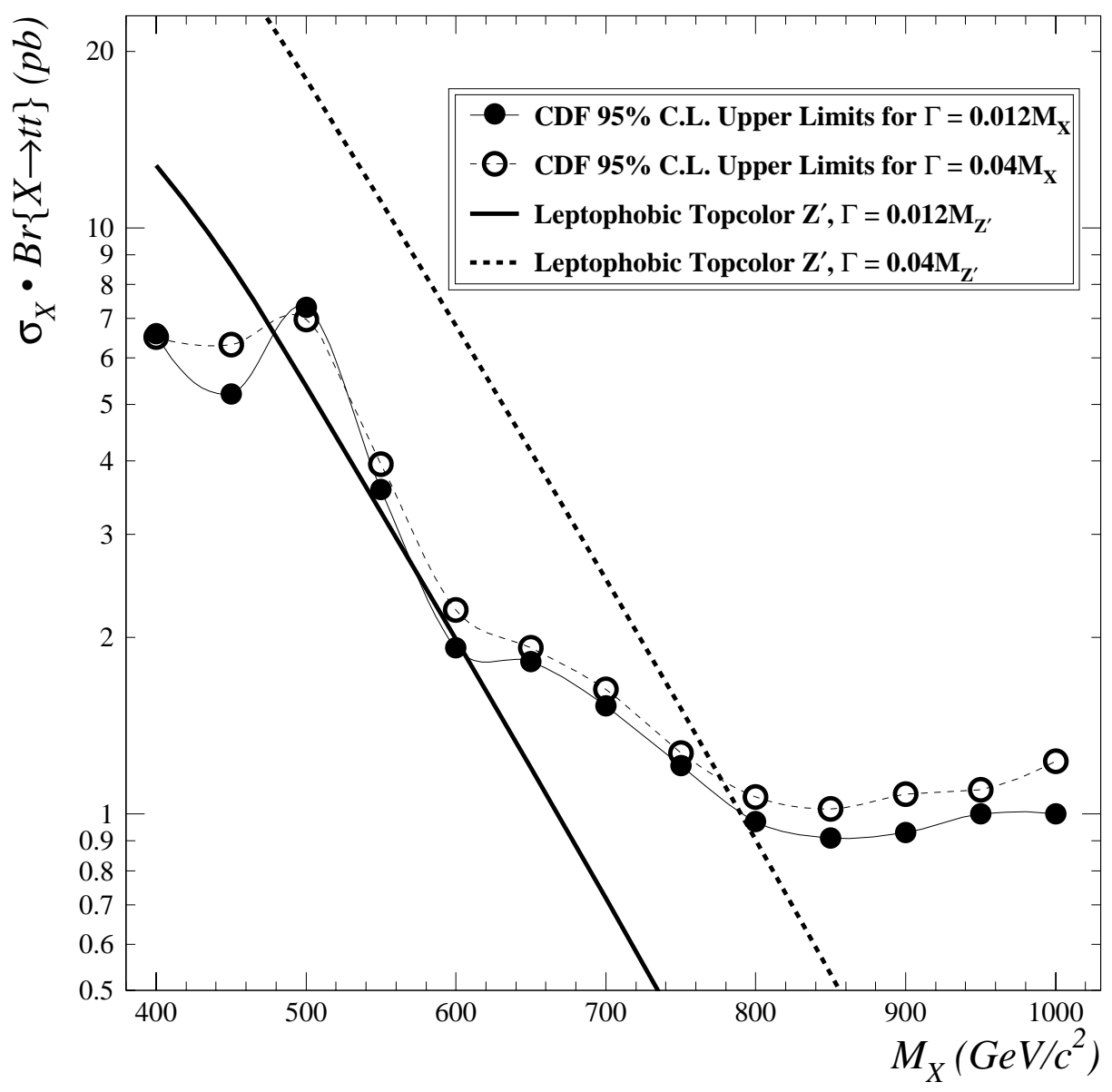

Figure 8: Cross-section limits for a narrow resonance decaying to $t \bar{t}[32]$ and expected cross section for a leptophobic topcolor $Z^{\prime}$ boson.

arise predominantly from "topcolor," a new QCD-like interaction which couples strongly to the third generation of quarks. An additional, strong, U(1) interaction (giving rise to a topcolor $\left.Z^{\prime}\right)$ precludes the formation of a $b$-quark condensate. 
CDF has searched [31] for the "topgluon," a massive coloroctet vector which couples preferentially to the third generation, in the mode $p \bar{p} \rightarrow g_{t} \rightarrow b \bar{b}$. The results are shown in Fig. 7 . Topgluon masses from approximately 0.3 to $0.6 \mathrm{TeV}$ are excluded at $95 \%$ confidence level, for topgluon widths in the range $0.3 m_{g_{t}}<\Gamma<0.7 m_{g_{t}}$. Results have also been reported by $\mathrm{CDF}[32]$ on a search for narrow resonances in the $t \bar{t}$ invariant mass distribution. The cross section limit is shown in Fig. 8 and excludes a leptophobic topcolor $Z^{\prime}$ with masses less than 480 (780) $\mathrm{GeV} / c^{2}$, for the case where its width $\Gamma=0.012(0.04) m_{Z^{\prime}}$. (DØ has carried out a similar search, with greater sensitivity [33], but has not derived comparable $Z^{\prime}$ mass limits.) A broad topgluon could also be detected in the same final state, though no results are yet available. In Run II, the Tevatron [24] should be sensitive to topgluon and topcolor $Z^{\prime}$ masses up to of order $1 \mathrm{TeV}$ in $b \bar{b}$ and $t \bar{t}$ final states. A detailed theoretical analysis of $B-\bar{B}$ mixing and light quark mass generation in top-color assisted technicolor shows that, at least in some models, the topgluon and $Z^{\prime}$ boson masses must be greater than about $5 \mathrm{TeV}$ [34].

The top-quark seesaw model of electroweak symmetry breaking [35] is a variant of the original top-condensate idea which reconciles top-condensation with a lighter top-quark mass. Such a model can easily be consistent with precision electroweak tests, either because the spectrum includes a light composite Higgs [36] or because additional interactions allow for a heavier Higgs [37]. Such theories may arise naturally from gauge fields propagating in compact extra spatial dimensions [38].

A variant of topcolor-assisted technicolor is flavor-universal, in which the topcolor $\mathrm{SU}(3)$ gauge bosons, called colorons,

HTTP://PDG.LBL.GOV Page $15 \quad$ Created: 6/13/2002 13:08 
couple equally to all quarks [39]. Flavor-universal versions of the seesaw model [40] incorporating a gauged flavor symmetry are also possible. In these models all left-handed quarks (and possibly leptons as well) participate in electroweak symmetrybreaking condensates with separate (one for each flavor) righthanded weak singlets, and the different fermion masses arise by adjusting the parameters which control the mixing of each fermion with the corresponding condensate.

A prediction of these flavor-universal models, is the existence of new heavy gauge bosons, coupling to color or flavor, at relatively low mass scales. The absence of an excess of high- $E_{T}$ jets in D $\varnothing$ data [41] has been used to constrain strongly-coupled flavor-universal colorons (massive color-octet bosons coupling to all quarks). A mass limit of between 0.8 and $3.5 \mathrm{TeV}$ is set [42] depending on the coloron-gluon mixing angle. Precision electroweak measurements constrain [43] the masses of these new gauge bosons to be greater than $1-3 \mathrm{TeV}$ in a variety of models, for strong couplings.

\section{Acknowledgments}

We thank Tom Appelquist, Bogdan Dobrescu, Robert Harris, Chris Hill, Greg Landsberg, Kenneth Lane, and Elizabeth Simmons for help in the preparation of this article. This work was supported in part by the Department of Energy under grant DE-FG02-91ER40676.

\section{References}

1. S. Weinberg, Phys. Rev. D19, 1277 (1979).

2. L. Susskind, Phys. Rev. D20, 2619 (1979).

3. For recent reviews, see K. Lane, hep-ph/0007304; R.S. Chivukula, hep-ph/9803219.

4. E. Eichten and K. Lane, Phys. Lett. 90B, 125 (1980).

HTTP://PDG.LBL.GOV Page $16 \quad$ Created: 6/13/2002 13:08 
5. For reviews, see E. Farhi and L. Susskind, Phys. Reports 74, 277 (1981);

R.K. Kaul, Rev. Mod. Phys. 55, 449 (1983);

R.S. Chivukula et al., hep-ph/9503202.

6. S. Dimopoulos, S. Raby, and G.L. Kane, Nucl. Phys. B182, 77 (1981).

7. S. Dimopoulos and L. Susskind, Nucl. Phys. B155, 237 (1979).

8. B. Holdom, Phys. Rev. D24, 1441 (1981) and Phys. Lett. 150B, 301 (1985);

K. Yamawaki, M. Bando, and K. Matumoto, Phys. Rev. Lett. 56, 1335 (1986);

T.W. Appelquist, D. Karabali, and L.C.R. Wijewardhana, Phys. Rev. Lett. 57, 957 (1986);

T. Appelquist and L.C.R. Wijewardhana, Phys. Rev. D35, 774 (1987) and Phys. Rev. D36, 568 (1987).

9. E. Eichten and K. Lane, Phys. Lett. B222, 274 (1989).

10. See review by Langacker and Erler, this volume.

11. E. Eichten, I. Hinchliffe, K.D. Lane, and C. Quigg, Rev. Mod. Phys. 56, 579 (1984) and Phys. Rev. D34, 1547 (1986).

12. K. Lane, Phys. Rev. D60, 075007 (1999).

13. S. Mrenna, Technihadron production and decay at LEP2, Phys. Lett. B461, 352 (1999).

14. E. Eichten, K. Lane, and J. Womersley, Phys. Lett. B405, 305 (1997).

15. CDF Collaboration (T. Affolder et al.), Phys. Rev. Lett. 84, 1110 (2000).

16. T. Sjostrand, Comp. Phys. Comm. 82, 74 (1994).

17. CDF Collaboration (F. Abe et al.), Phys. Rev. Lett. 83, 3124 (1999).

18. D $\varnothing$ Collaboration (V.M. Abazov et al.), Phys. Rev. Lett. 87, 061802 (2001).

19. CDF Collaboration, K. Maeshima, presented at the Workshop on New Strong Dynamics at Run II, Fermilab (October 1998).

HTTP://PDG.LBL.GOV Page $17 \quad$ Created: 6/13/2002 13:08 
20. DELPHI Collaboration, Note 2001-086 Conf 514, July 2001 presented at Lepton Photon (2001).

21. L3 Note 2428, June 1999, submitted to the International Europhysics Conference on High Energy Physics, Tampere, Finland (July 1999).

22. K. Lane and M.V. Ramana, Phys. Rev. D44, 2678 (1991).

23. CDF Collaboration (F. Abe et al.), Phys. Rev. D55, R5263 (1997).

24. K. Cheung and R.M. Harris, hep-ph/9610382.

25. CDF Collaboration (F. Abe et al.), Phys. Rev. Lett. 82, 3206 (1999).

26. CDF Collaboration (T. Affolder et al.), Phys. Rev. Lett. 85, 2056 (2000).

27. A. Zerwekh and R. Rosenfeld, Phys. Lett. B503, 325 (2001);

R.S. Chivukula, et al., Boston Univpreprint BUHEP-0119.

28. V.A. Miransky, M. Tanabashi, and K. Yamawaki, Phys. Lett. 221B, 177 (1989) and Mod. Phys. Lett. 4, 1043 (1989);

Y. Nambu, EFI-89-08 (1989);

W.J. Marciano, Phys. Rev. Lett. 62, 2793 (1989).

29. W.A. Bardeen, C.T. Hill, and M. Lindner, Phys. Rev. D41, 1647 (1990).

30. C.T. Hill, Phys. Lett. B345, 483 (1995);

see also Phys. Lett. 266B, 419 (1991).

31. CDF Collaboration (F. Abe et al.), Phys. Rev. Lett. 82, 2038 (1999).

32. CDF Collaboration (F. Affolder et al.), Phys. Rev. Lett. 85, 2062 (2000).

33. D0 Collaboration (V. Abazov et al.), Phys. Rev. Lett. 87, 231801 (2001).

34. G. Burdman et al., Phys. Lett. B514, 41 (2001).

35. B.A. Dobrescu and C.T. Hill, Phys. Rev. Lett. 81, 2634 (1998).

HTTP://PDG.LBL.GOV Page $18 \quad$ Created: 6/13/2002 13:08 
36. R.S. Chivukula et al., Phys. Rev. D59, 075003 (1999).

37. H. Collins, A. Grant, and H. Georgi, Phys. Rev. D61, 055002 (2000).

38. B.A. Dobrescu, Phys. Lett. B461, 99 (1999);

H.-C. Cheng, et al., Nucl. Phys. B589, 249 (2000).

39. E.H. Simmons, Nucl. Phys. B324, 315 (1989).

40. G. Burdman and N. Evans, Phys. Rev. D59, 115005 (1999).

41. DØ Collaboration (B. Abbott et al.), Phys. Rev. Lett. 82, 2457 (1999).

42. I. Bertram and E.H. Simmons, Phys. Lett. B443, 347 (1998).

43. G. Burdman, R.S. Chivukula, and N. Evans, Phys. Rev. D61, 035009 (2000).

\section{MASS LIMITS for Resonances \\ in Models of Dynamical Electroweak Symmetry Breaking}

$\operatorname{VALUE}(\mathrm{GeV})$

CL\% DOCUMENT ID

TECN COMMENT

- - We do not use the following data for averages, fits, limits, etc.

\begin{tabular}{|c|c|c|c|c|}
\hline$>207$ & 95 & ${ }^{1}$ ABAZOV & 01B D0 & $\rho_{T} \rightarrow e^{+} e^{-}$ \\
\hline none 90-206.7 & 95 & ${ }^{2}$ ABDALLAH & $01 \mathrm{DLPH}$ & $e^{+} e^{-} \rightarrow \rho_{T}$ \\
\hline & & ${ }^{3}$ AFFOLDER & OOF CDF & $\begin{array}{l}\text { color-singlet techni- } \rho \text {, } \\
\qquad \rho_{T} \rightarrow W \pi_{T}, 2 \pi_{T}\end{array}$ \\
\hline$>600$ & 95 & 4 AFFOLDER & $00 \mathrm{~K} C D F$ & $\begin{array}{l}\text { color-octet techni- } \rho \text {, } \\
\quad \rho_{T 8} \rightarrow 2 \pi_{L Q}\end{array}$ \\
\hline$>480$ & 95 & ${ }^{5}$ AFFOLDER & $00 \mathrm{~L} C D F$ & top-color $Z^{\prime}$ \\
\hline none $350-440$ & 95 & ${ }^{6} \mathrm{ABE}$ & 99F CDF & $\begin{array}{l}\text { color-octet techni- } \rho \text {, } \\
\qquad \rho_{T 8} \rightarrow \bar{b} b\end{array}$ \\
\hline$>465$ & 95 & ${ }^{7} \mathrm{ABE}$ & $99 \mathrm{H} \mathrm{CDF}$ & $\begin{array}{l}\text { color-octet techni- } \rho \text {, } \\
\quad \rho_{T 8} \rightarrow 2 \pi_{L Q}\end{array}$ \\
\hline & & $8 \mathrm{ABE}$ & 99N CDF & techni- $\omega, \omega_{T} \rightarrow \gamma \bar{b} b$ \\
\hline none $260-480$ & 95 & ${ }^{9} \mathrm{ABE}$ & 97G CDF & $\begin{array}{l}\text { color-octet techni- } \rho \text {, } \\
\quad \rho_{T 8} \rightarrow 2 \text { jets }\end{array}$ \\
\hline
\end{tabular}

${ }^{1}$ ABAZOV 01B searches for vector techni-resonances $\left(\rho_{T}, \omega_{T}\right)$ decaying to $e^{+} e^{-}$. The limit assumes $M_{\rho_{T}}=M_{\omega_{T}}<M_{\pi_{T}}+M_{W}$.

2 The limit is independent of the $\pi_{T}$ mass. See their Fig. 9 and Fig. 10 for the exclusion plot in the $M_{\rho_{T}}-M_{\pi_{T}}$ plane. ABDALLAH 01 limit on the techni-pion mass is $M_{\pi_{T}}>79.8$ $\mathrm{GeV}$ for $N_{D}=2$, assuming its point-like coupling to gauge bosons.

${ }^{3}$ AFFOLDER 00F search for $\rho_{T}$ decaying into $W \pi_{T}$ or $\pi_{T} \pi_{T}$ with $W \rightarrow \ell \nu$ and $\pi_{T} \rightarrow$ $\bar{b} b, \bar{b} c$. See Fig. 1 in the above Note on "Dynamical Electroweak Symmetry Breaking" for the exclusion plot in the $M_{\rho_{T}}-M_{\pi_{T}}$ plane.

HTTP://PDG.LBL.GOV Page $19 \quad$ Created: 6/13/2002 13:08 
${ }^{4}$ AFFOLDER 00K search for the $\rho_{T 8}$ decaying into $\pi_{L Q} \pi_{L Q}$ with $\pi_{L Q} \rightarrow b \nu$. For $\pi_{L Q} \rightarrow c \nu$, the limit is $M_{\rho_{T 8}}>510 \mathrm{GeV}$. See their Fig. 2 and Fig. 3 for the exclusion plot in the $M_{\rho_{T 8}}-M_{\pi_{L Q}}$ plane.

${ }^{5}$ AFFOLDER 00L search for top-color $Z_{\text {top }}^{\prime}$ decaying into $\bar{t} t$. The quoted limit is for $Z_{\text {top }}^{\prime}$ with decay width $\Gamma=0.012 M_{Z^{\prime}}$. For $\Gamma=0.04 M_{Z^{\prime}}$, the limit becomes $780 \mathrm{GeV}$.

${ }^{6} \mathrm{ABE} 99 \mathrm{~F}$ search for a new particle $X$ decaying into $b \bar{b}$ in $p \bar{p}$ collisions at $E_{\mathrm{cm}}=1.8 \mathrm{TeV}$. See Fig. 7 in the above Note on "Dynamical Electroweak Symmetry Breaking" for the upper limit on $\sigma(p \bar{p} \rightarrow X) \times \mathrm{B}(X \rightarrow b \bar{b})$. ABE 99F also exclude top gluons of width $\Gamma=0.3 M$ in the mass interval $280<M<670 \mathrm{GeV}$, of width $\Gamma=0.5 \mathrm{M}$ in the mass interval $340<M<640 \mathrm{GeV}$, and of width $\Gamma=0.7 M$ in the mass interval $375<M<560 \mathrm{GeV}$.

$7 \mathrm{ABE} 99 \mathrm{H}$ search for the color-octet techni- $\rho$ decaying into a pair of color-triplet technipions which subsequently decay into $\tau+$ jet. See Fig. 6 in the above Note on "Dynamical Electroweak Symmetry Breaking" for the exclusion plot in the $M_{\rho_{T 8}}-M_{\pi_{L Q}}$ plane.

${ }^{8} \mathrm{ABE} 99 \mathrm{~N}$ search for the techni- $\omega$ decaying into $\gamma \pi_{T}$. The technipion is assumed to decay $\pi_{T} \rightarrow b \bar{b}$. See Fig. 2 in the above Note on "Dynamical Electroweak Symmetry Breaking" for the exclusion plot in the $M_{\omega_{T}}-M_{\pi_{T}}$ plane.

${ }^{9} \mathrm{ABE} 97 \mathrm{G}$ search for a new particle $X$ decaying into dijets in $p \bar{p}$ collisions at $E_{\mathrm{cm}}=1.8$ TeV. See Fig. 5 in the above Note on "Dynamical Electroweak Symmetry Breaking" for the upper limit on $\sigma(p \bar{p} \rightarrow X) \times \mathrm{B}(X \rightarrow 2 j)$.

\section{REFERENCES FOR Technicolor}

\begin{tabular}{|c|c|c|c|c|}
\hline ABAZOV & 01B & PRL 87061802 & V.M. Abazov et al. & (D0 Collab.) \\
\hline ABDALLAH & 01 & EPJ C22 17 & J. Abdallah et al. & (DELPHI Collab.) \\
\hline AFFOLDER & $00 \mathrm{~F}$ & PRL 841110 & T. Affolder et al. & (CDF Collab.) \\
\hline AFFOLDER & $00 \mathrm{~K}$ & PRL 852056 & T. Affolder et al. & (CDF Collab.) \\
\hline AFFOLDER & $00 \mathrm{~L}$ & PRL 852062 & T. Affolder et al. & (CDF Collab.) \\
\hline $\mathrm{ABE}$ & $99 F$ & PRL 822038 & F. Abe et al. & (CDF Collab.) \\
\hline $\mathrm{ABE}$ & $99 \mathrm{H}$ & PRL 823206 & F. Abe et al. & (CDF Collab.) \\
\hline ABE & $99 N$ & PRL 833124 & F. Abe et al. & (CDF Collab.) \\
\hline ABE & $97 G$ & PR D55 R5263 & F. Abe et al. & (CDF Collab.) \\
\hline
\end{tabular}

HTTP://PDG.LBL.GOV Page $20 \quad$ Created: 6/13/2002 13:08 